\title{
Coronavirus: Discover the Structure of Global Knowledge, Hidden Patterns \& Emerging Events
}

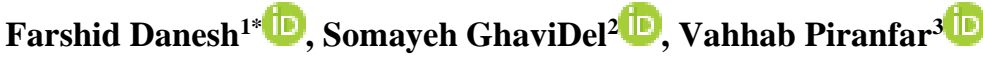

1. Information Management Research Department, Regional Information Center for Science and Technology (RICeST), Shiraz, Iran

2. Department of Knowledge and Information Science, Kharazmi University, Tehran, Iran

3. Research and Development Department, Farname Inc, Thornhill, Canada

\section{Article Info \\ 10.30699/jambs.28.130.253 \\ Received: 2020/07/12 \\ Accepted: 2020/09/05; \\ Published Online: 30 Sep 2020; \\ Use your device to scan and read the article online

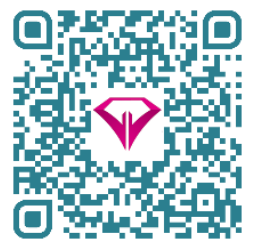

\begin{abstract}
Corresponding Information: Farshid Danesh,

Information Management Research Department, Regional Information Center for Science and Technology
\end{abstract} (RICeST), Shiraz, Iran \\ E-Mail: \\ farshiddanesh@ricest.ac.ir}

\section{ABSTRACT}

Background \& Objective: The present study aimed at exploring the structure of global knowledge, hidden patterns, and emerging Coronavirus events using co-word techniques. Co-word analysis is one of the most efficient scientific methods to analyze the structure and dynamics of knowledge and the general state of research.

Materials \& Methods: This applied research performed using Co-word analysis. The statistical population is 4102 keywords from Web of Science Core Collection indexed documents on Coronavirus retrieved through advanced search (1970-2019). To identify the keywords used to design a search strategy, the Medical Subject Heading browser was utilized. After the keyword editing process, the threshold identified, and UCINET, VOSviewe, and SPSS 16 were used to analyze the data.

Results: The highest frequent keyword was "Severe Acute Respiratory Syndrome (SARS)" with a frequency of 276. Nineteen subject clusters were the result of a hierarchical clustering analysis by the Wards' method. Clusters 4 and 15 were the biggest ones with nine keywords. Strategic diagram analysis showed that the most prominent Coronavirus clusters' most prominent clusters are in Quadrant III of the strategic diagram.

Conclusion: The results showed that Coronavirus research's intellectual structure in the form of 19-topic thematic clusters and determining the degree of cluster cohesion makes it possible to discover complex conceptual relationships of valid international Coronavirus research. The results of this paper could also be used to guide medical researchers, especially coronavirus scientists. Medical policymakers can also more effectively present strategic plans by becoming aware of the global knowledge structure, hidden patterns, and emerging international coronavirus events.

Keywords: Coronavirus; COVID-19; 2019-nCoV; Coronavirus Disease 2019; Co-word Analysis; Scientometrics; Strategic diagram

\section{Introduction}

COVID-19 is the name of a new Coronavirus that has been identified. The World Health Organization (WHO) has identified the outbreak of the emerging COVID-19 virus as a public health emergency at the international level, and also, with the awareness of spreading the disease, 2019-nCoV is considered a pandemic disease (1-3). Infectious diseases have always been a serious threat to humankind, especially health care providers (4). Coronaviruses (CoVs) are the largest group of viruses in the genus Nidovirales, which includes families of Coronaviridae, Arteriviridae, and Roniviridae. Coronaviridae is a family of Envelopes, non-segmented, and RNApositive single-stranded viruses $(5,6)$. Coronaviruses cause acute respiratory diseases and infections in humans and various diseases in mammals (7-9). Today, COVID-19, a species belonging to the Coronavirinae subfamily in the Coronaviridae family, a new dangerous member of these crowned viruses, has the highest mortality rate $(3,10)$. The challenging and global spread of COVID-19 outbreaks in China since January 2020 and the subsequent globalization of the virus has prompted medical researchers to seek new ways to prevent, produce vaccines, medicines, and treat COVID19. So various and extensive researchers have conducted researches and published the results as scholarly documents in peer-reviewed journals. Some of these are high-ranked journals also indexed in the world's most prestigious and oldest citation database, Web of Science (11). 
Discovering the structure of global knowledge, hidden and visible patterns, as well as emerging Coronavirus events, can provide practical information to medical researchers. Therefore, scientometric studies and content analysis of prestigious publications indexed in citation databases such as the Web of Science and providing essential and categorized findings in the field of Coronavirus are some of the critical issues and challenges. Therefore, it is necessary to conduct and publish scientometric studies' applied results at the international level. Scientific studies can represent scientific productions and show gaps in scientific fields (12).

Evidence suggests that medical researchers need to be aware of Coronavirus scientometrics studies; therefore, studying the structure of knowledge and analyzing the relationships between concepts and thematic clusters of Coronavirus publications with phonological and cluster analysis methods is very important $(13,14)$. Co-word analysis examines the main subjects, semantic structure, and evolution of the works of the transition of time (15). It provides the possibility of visualizing the conceptual structure of scientific disciplines (16). The COVID-19 study results, which affect many people worldwide every day and, in most cases, leads to death, are essential (17). Studies have shown that a few scientiometric studies on Coronavirus have been published and used by researchers in various medical sciences fields to date. These studies are discussed below.

Danesh \& Ghavidel performed a scientometrics analysis on the 50 years global scientific collection of Coronavirus indexed in the WOS from 1970 to 2020 (17). This study showed that the highest number of science production was found in 2005, and the highest citation number was recorded in 2019. "Enjuanes L.", the United States, and the University of Hong Kong were shown as the most proliferated author, the most productive country, and the top organization in Coronavirus in the last half-century, respectively. Bonilla-Aldana et al. conducted a scientific bibliometric analysis on coronaviruses (MERS-CoV, SARS-CoV, and nCoV2019) from biomedical journals indexed in the WOS, PubMed, and Scopus databases, using the term "coronavirus" as the primary keyword from January 1951 to January 2020 (18). This study showed that the USA and China performed primary and fundamental roles in CoV research. The USA was a leader in scientific production, with almost a third of the articles. Then Asia, Hong Kong, and South Korea were among the top ten records. Danesh \& Ghavidel also analyzed the Human Papilloma Virus based on the Co-Word "Analysis" and "strategic diagram" 17278 keywords of 13249 articles in the HPV area during 2014-2018 (19). The keyword "Cervical Cancer" had the highest frequency, and along with "Cervical Intraepithelial Neoplasia," they were co-word couples. The most clusters in HPV were placed in quadrant III of the strategic diagram, which means these clusters of subjects were emerging or declining. Singh et al. examined the scientometric analyses of the Nipah virus's scientific outcome from Scopus in 2018 (20). The predominant topics of 373 journals discussed in the papers included: characterization, clinical symptoms, pathogenesis, transmission, outbreaks, and vaccine developments. Zyoud investigated the bibliometric analysis of 883 MERS-CoV publications in the Scopus from 2012 to 2015 from 92 countries/territories. The USA was the most significant contributor, with 319 published articles. The h-index for the MERS-CoV-associated publications was recorded at $48(21)$. The highest h-index was related to the USA, Kingdom of Saudi Arabia (KSA), and the UK. The Netherlands produced the highest number of publications with international research collaborations. Chiu et al. studied the bibliometric analysis of the SARS-related publications in the WOS in the early stage in 2003 (22). The USA had the highest production by $30 \%$ of the total, closely followed by Hong Kong with $24 \%$. The main countries published $63 \%$ of publications. Singh (2016) investigated through the Scopus scientometric analysis of 573 researches on the Zika virus. The results revealed a high degree of research/authorship collaborations on Zika virus (20).

Reviewing the literature suggests that no extensive scientific research has been conducted in this area despite Coronavirus's strategic importance. Among the published articles, no comprehensive and analytical study of Coronavirus was found using the co-word "analysis method". Conducting the present research looks vital, given the importance of the subject and the lack of scientometrics information using the co-occurrence technique of Coronavirus. This paper's main issue is to apply co-word "analysis" as one of the conventional methods in scientometrics to identify the logical structure and visualization of the coronavirus conceptual network in the world. This paper's outcome contributes to the purposefulness of the research, planning, and policymaking at the national and international levels. Besides, it is useful for identifying the appropriate treatment goals and better decision-making, as well as for frontline and leaders of health professionals and travel medicine practitioners to achieve more efficient solutions in the shortest possible time.

This study's outcome can also play a crucial role in the correct and authentic allocation of budgets (23). Alongside this research's strategic importance, it is necessary to perform and publish its results at the international level. The hierarchical clustering analysis is necessary to achieve the primary goal of this research, clustering coronavirus subjects in the world and discovering researchers' intellectual structure based on Coronavirus results. It is also necessary to identify the degree of clusters coherence in the strategic diagram using the method of "co-occurrences of words". The discovery of global knowledge structure, hidden patterns, and emerging coronavirus events using the co-word "analysis" is the main issue of this research. 


\section{Materials and Methods}

The method used in this paper is a co-word analysis. Co-word analysis technique, based on the calculation of the rate of all co-occurrence of words, provides the possibility of visualizing the structure of subjects and concepts (24). This method is suitable for detecting communications, tracking structural changes, and developing the conceptual, social network, and essential links (25). Therefore, this method was used to discover the structure of global knowledge, hidden patterns, and emerging Coronavirus events.

The statistical population is all English language articles published about Coronavirus from 1970 to 2019. Using this interval can comprehensively show the intellectual structure of Coronavirus. The Web of Science Core Collection (WOSCC) was utilized to access the research community. It is the world's most prestigious, widely used, and the oldest citation database (11). Since 1900, with full cited reference indexing and, in comparison with other databases, Web of Science has provided more valuable and comprehensive documents for downloading in various scientific disciplines (26). The MeSH (Medical Subject Headings) browser is used to identify the Coronavirus main keywords. After consulting with experts, the search strategy was designed and developed in the Web of Science advanced search. The search strategy is as follows.

\section{"Coronavirus" OR "ALPHACORONAVIRUS" OR "BETACORONAVIRUS" OR "DELTACORONAVIRUS" OR \\ "GAMMACORONAVIRUS" OR "Bulbul coronavirus HKU11" OR "Coronavirus HKU15" OR "Munia coronavirus HKU13" OR "Rabbit Coronavirus" OR \\ "Thrush coronavirus HKU12".}

The advanced search results using the Title Tag (TI) were limited to ARTICLES, MEETING ABSTRACT, PROCEEDINGS PAPER, and REVIEW in terms of the document type. In terms of language, retrieved documents were limited to the English language for the period 1970-2019. The reason for choosing the period 1970-2019 was the lack of any publications about Coronavirus prior to 1970 . A total of 4102 Keywords extracted from indexed documents were retrieved through advanced search. The keywords were studied in a process that included editing, integrating, deleting, and mending. After reviewing the process, the number of keywords decreased to 4058. Ravar Matrix: Ravar PreMap was used to refine the data and create a cooccurrence matrix.

To prepare the matrix for co-word analysis, researchers selected the threshold. After performing some tests, Bradford's law was utilized in the final analysis. In previous co-word analysis studies, different thresholds were used to include the top keywords in the final analysis $(13,27)$. The threshold of this article determined using the Bradford Law on 11 ; in other words, keywords that have been repeated more than ten times. Using the threshold, 86 highfrequency keywords were identified and analyzed in the co-word analysis (Table 1). To perform the co-word analysis, hierarchical clustering was done with the Ward method and Squared Euclidean distance calculation using SPSS 16 (SPSS Inc., Chicago, Ill., USA). Then, the dendrogram diagram was drawn with 19 subject clusters (Figure 2 and Table 2). The use of the Ward method for hierarchical clustering analysis has been performed in many previous co-word analysis' studies (28-32).

Subsequently, the co-word matrix characteristics, such as Centrality and Density, were analyzed using the UCINET software to obtain more information about the Coronavirus's co-word structure. If the node has many relationships with other nodes in a network, it has a higher centrality and an important place. Density means more cohesion and more internal interconnection between nodes. Density is a research area that demonstrates its ability to sustain and develop. The Centrality and Density of each of the subject clusters can be demonstrated in the form of a Strategic Diagram, revealing the Coronavirus's state and evolutionary trends. The Strategic Diagram's horizontal axis was Degree / Centrality, and its vertical axis was density $(27,32)$. In Strategic Diagram, the higher the centrality of a cluster is, the more critical that cluster is in the research topic, and the higher the density of a thematic cluster is, the more capable or mature that cluster is. Therefore, in a strategic chart, the $\mathrm{X}$-axis represents the centrality, and the $\mathrm{Y}$-axis represents the Density (Figure 3). The Strategic Diagram can be illustrated in 4 quadrants, each with different densities and centralities, and each cluster in each quadrant has a different status. Finally, a visualization was performed using VOSviewer1.6.11.

\section{Results}

\section{Coronavirus Top Keywords Based on Co-word Analysis}

According to Table 1, the top 20 most frequently keywords in the Coronavirus subject area were presented based on the co-word analysis. The "Severe Acute Respiratory Syndrome (SARS)," with 276 frequency, had the highest frequency among all keywords. MERS-COV and SARS-COV were also ranked second and third with a frequency of 265 and 259 , respectively. Table 1 showed the importance of these keywords for researchers at this time. 
Table 1. Ranking the top 20 keywords in the Coronavirus (1970-2019)

\begin{tabular}{|c|c|c|}
\hline Rank & Keywords & Frequency \\
\hline 1 & Severe Acute Respiratory Syndrome (SARS) & 276 \\
\hline 2 & The Middle East Respiratory Syndrome Coronavirus (MERS-COV) & 265 \\
\hline 3 & Severe Acute Respiratory Syndrome CORONAVIRUS (SARS-COV) & 259 \\
\hline 4 & Middle East Respiratory Syndrome & 97 \\
\hline 5 & Spike Protein & 68 \\
\hline 6 & Bovine Coronavirus (BCOV) & 65 \\
\hline 7 & Infectious bronchitis virus (IBV) & 58 \\
\hline 8 & Porcine Delta Coronavirus (PDCOV) & 58 \\
\hline 9 & Nucleocapsid protein & 55 \\
\hline 10 & Feline Coronavirus (FCOV) & 54 \\
\hline 11 & Phylogenetic analysis & 53 \\
\hline 12 & Epidemiology & 49 \\
\hline 13 & Vaccine & 46 \\
\hline 14 & Human Coronavirus & 45 \\
\hline 15 & Enzyme-linked Immunosorbent Assay (ELISA) & 44 \\
\hline 16 & Transmissible gastroenteritis virus (TGEV) & 42 \\
\hline 17 & Real-time RT-PCR & 40 \\
\hline 18 & Canine Coronavirus $(\mathrm{CCoV})$ & 38 \\
\hline 19 & Antibodies & 37 \\
\hline 20 & Feline infectious peritonitis (FIP) & 37 \\
\hline
\end{tabular}

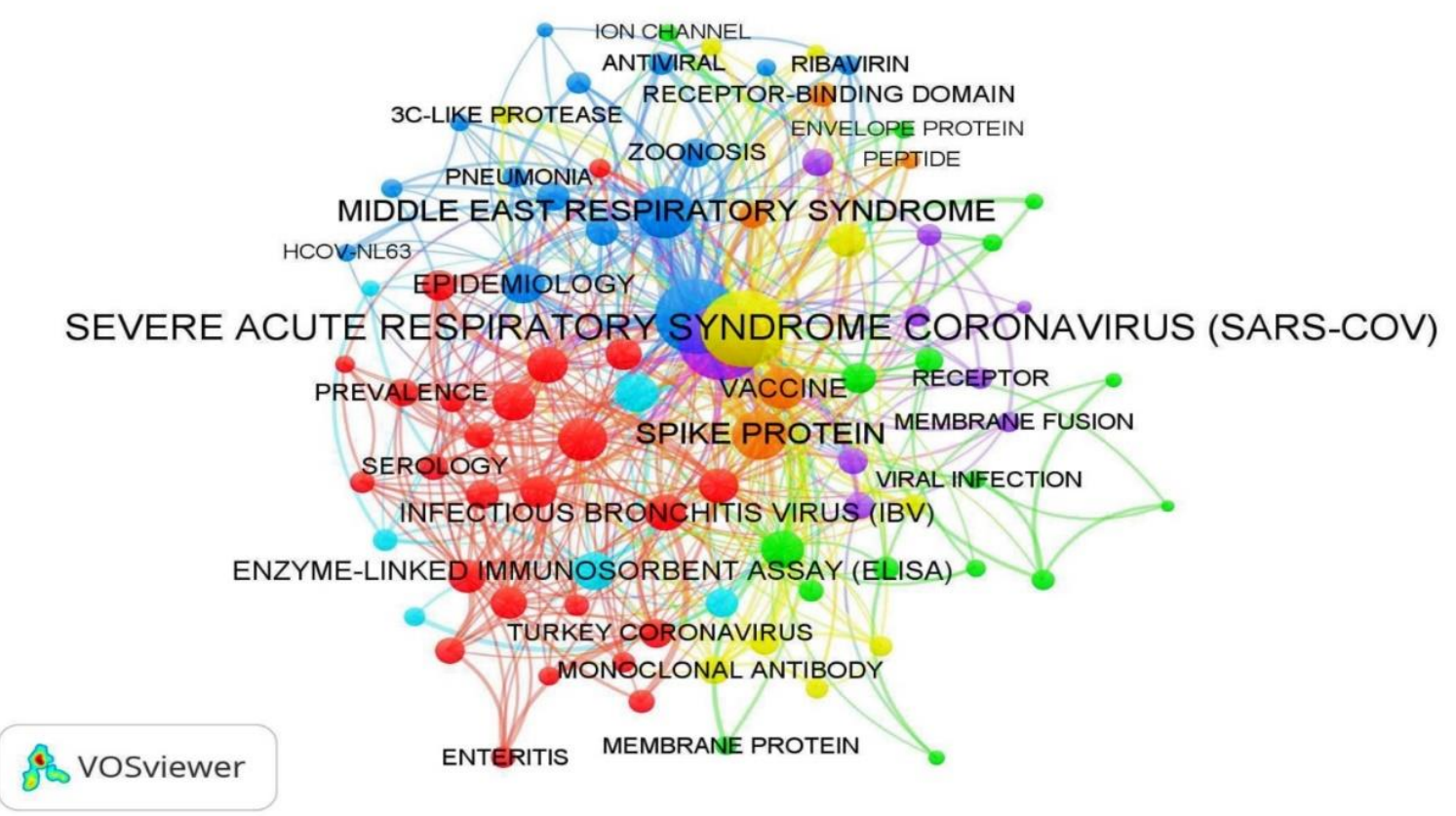

Figure 1. Coronavirus High-frequency Keywords network structure 
Figure 1 shows the high frequency keywords in Coronavirus subject area. Hot topics were based on the high frequent Coronavirus related keywords, including 86 keywords with a frequency above 10 . These subjects were shown in the form of a network with VOSviewer in Figure 1. The size of the nodes indicates the weight of the scientific publications of each subject. The colors represent the formed nodes. In the map drawn with VOSviewer, the topics were networked according to existing algorithms. The colors indicate the subjects, and the diameter of the lines indicates the connection between the nodes. Hot subjects with higher cooccurrence are centered on the network, and topics with lower co-occurrence are around the network.

\section{Coronavirus Hierarchical Clustering in the World}

Cluster analysis of scientific publications in the last half-century has led to 19 clusters in Coronavirus. Table 2 also shows the Coronavirus clusters along with the keywords for each cluster.

Table 2. Coronavirus subject clusters based on clustering analysis

\begin{tabular}{|c|c|c|}
\hline Cluster & Keywords & $\begin{array}{l}\text { Number of } \\
\text { Keywords }\end{array}$ \\
\hline Cluster 1 & Feline Coronavirus (FCOV); Feline Infectious Peritonitis (FIP) & 2 \\
\hline Cluster 2 & Transmissible Gastroenteritis Virus (TGEV); Porcine Respiratory Coronavirus (PRCV) & 2 \\
\hline Cluster 3 & $\begin{array}{c}\text { Bovine Coronavirus (BCOV); Turkey Coronavirus; Diarrhea; Enteritis; } \\
\text { Immunohistochemistry (IHC) }\end{array}$ & 5 \\
\hline Cluster 4 & $\begin{array}{c}\text { Enzyme-linked Immunosorbent Assay (ELISA); Real-time RT-PCR; Canine } \\
\text { Coronavirus (CCoV); Antibodies; Diagnosis; Polymerase Chain Reaction (PCR); } \\
\text { Serology; Equine MERS-COV; Seroprevalence }\end{array}$ & 9 \\
\hline Cluster 5 & Infectious bronchitis virus (IBV); Avian Coronavirus & 2 \\
\hline Cluster 6 & Porcine Delta Coronavirus (PDCOV); Phylogenetic Analysis; Prevalence & 3 \\
\hline Cluster 7 & Recombination; Genotype; Mutations; Genome; Molecular Epidemiology & 5 \\
\hline Cluster 8 & $\begin{array}{l}\text { Epidemiology; Human Coronavirus; Respiratory tract infection (RTI); HCoV-NL63; } \\
\text { Pneumonia; Human Coronavirus NL63; Respiratory Viruses }\end{array}$ & 7 \\
\hline Cluster 9 & Interferon; Antiviral; Ribavirin & 3 \\
\hline Cluster 10 & Spike Protein; Neutralizing Antibodies (NAbs); Receptor-Binding Domain (RBD) & 3 \\
\hline Cluster 11 & $\begin{array}{l}\text { Middle East Respiratory Syndrome Coronavirus (MERS-COV); Middle East } \\
\text { Respiratory Syndrome (MERS); Transmission; Zoonosis; Outbreak }\end{array}$ & 5 \\
\hline Cluster 12 & $\begin{array}{l}\text { Demyelination; Viral Infection; Multiple sclerosis (MS); Central Nervous System } \\
\text { (CNS) }\end{array}$ & 4 \\
\hline Cluster13 & Membrane fusion; Viral entry; Protease & 3 \\
\hline Cluster 14 & Envelope Protein; Ion channels & 2 \\
\hline Cluster 15 & $\begin{array}{l}\text { Severe Acute Respiratory Syndrome Coronavirus (SARS-COV); spike; Angiotensin- } \\
\text { converting enzyme } 2 \text { (ACE2); Nucleocapsid; Accessory Protein; Papain-like protease } \\
\text { (PLpro); 3C-like protease (3CLpro); Receptor; X-ray crystallography (XRC) }\end{array}$ & 9 \\
\hline Cluster 16 & $\begin{array}{c}\text { Virus ; Pathogenesis; Apoptosis; Pathogenicity; Inflammation; Viral replication; } \\
\text { Infection }\end{array}$ & 7 \\
\hline Cluster 17 & $\begin{array}{c}\text { Severe Acute Respiratory Syndrome (SARS); Vaccine; Spike glycoprotein; Reverse } \\
\text { genetics; Inhibitor; Peptide }\end{array}$ & 6 \\
\hline Cluster 18 & Monoclonal Antibody; Epitope & 2 \\
\hline Cluster 19 & $\begin{array}{c}\text { Nucleocapsid protein; Mouse Hepatitis Virus (MHV); Murine Coronavirus; Innate } \\
\text { immunity; Membrane protein; RNA virus }\end{array}$ & 6 \\
\hline
\end{tabular}




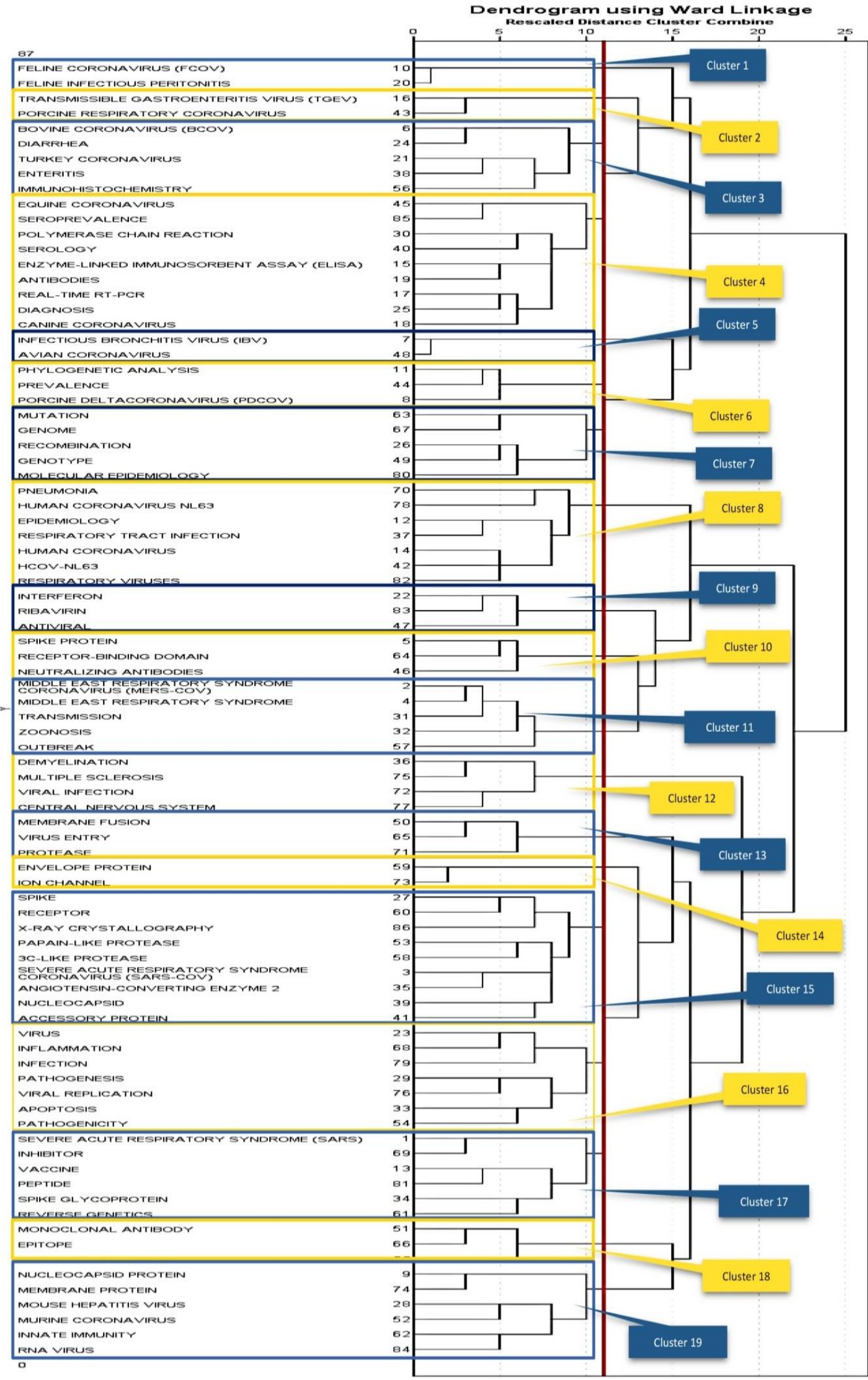

Figure 2. Coronavirus subject clustering dendrogram in the world (1970-2019) 
The First cluster has two keywords. The tenth high frequent keyword is "Feline Coronavirus (FCOV)," and "Feline infectious peritonitis (FIP)" is the twentieth high frequent keyword. They are located in the first cluster. Researchers have noted the importance of Coronavirus and the Peritonitis of infection. Transmissible Gastroenteritis Virus (TGEV) is the most frequent keyword in the second cluster. The keywords in the $2^{\text {nd }}$ cluster illustrate the issue of Gastroenteritis and the transmission of crowned Coronaviruse from animals; animals such as Porcine and Bovine. The third cluster has five keywords. Bovine Coronavirus (BCOV) is the sixth most frequent keyword in this research. According to the third cluster subjects' identification and analysis, "Turkey Bovine Coronavirus Immunohistochemistry" is the main subject of this cluster. The fourth cluster has the highest number of keywords among all clusters. It consists of nine keywords. Keywords for this cluster are "Enzymelinked Immunosorbent Assay (ELISA)", "Real-time RT-PCR", and "Canine coronavirus (CCoV)". Considering the presence of Polymerase Chain Reaction keywords and ELISA, Serology, and Realtime RT-PCR tests, it can say that the main topic of this cluster is "Coronavirus Laboratory Testing". The fifth cluster has two keywords. The seventh most frequent keyword (Infectious bronchitis virus" (IBV)) is in this cluster. The main subject of this cluster is the "Avian Coronavirus infectious bronchitis virus." The sixth cluster has three keywords. "Porcine Delta Coronavirus (PDCOV)" and "Phylogenetic analysis" are the eighth and the $11^{\text {th }}$ most frequent keyword in this cluster, respectively. The main subject of the $6^{\text {th }}$ cluster is the "prevalent phylogenetic analysis of porcine Coronavirus". Five keywords involved in the formation of the seventh cluster. Analysis of the subjects of this cluster showed that the main subject of this cluster is "Molecular Epidemiology of Recombination Human Coronavirus". The eighth cluster has seven keywords. Due to the presence of "Epidemiology" and "Human Coronavirus" in this cluster, the main subject is "Human Coronavirus and viral infections". The ninth cluster includes three keywords, Interferon and Antiviral, Ribavirin. So, the subject of this cluster is "Coronavirus Drug Therapy". Three keywords have been involved in the formation of the tenth cluster. "Spike Protein" is the fifth most frequent keyword with the rank of sixty-eight among all keywords in this research. The keywords in the tenth cluster indicate that "Coronavirus antibodies" have been the subject of studies of researchers. The eleventh cluster has five keywords. The second and the fourth most frequently keywords in this domain are "MERSCOV" and "Middle East Respiratory Syndrome". Based on these keywords, this cluster is about the "Outbreaks of zoonotic diseases caused by viruses MERS-COV". The $12^{\text {th }}$ cluster consists of four keywords. Coronavirus infection causes demyelination of the Central Nervous System (CNS). The keywords indicate that the subject of the $12^{\text {th }}$ cluster could be "Coronavirus infects in the CNS". This cluster has three keywords. Analyzing the subjects of the keywords of the $13^{\text {th }}$ cluster suggests that "Structures and Mechanisms of Coronavirus Cell" well illustrates the central theme of this cluster. "Envelope Protein" and "Ion channels" are the keywords of the fourteenth cluster. CoV envelope (E) protein is involved in the life cycle of the virus. According to the keywords in this cluster, the "CoV life cycle" indicated the subject of the $14^{\text {th }}$ cluster.

The cluster fifteen, like cluster four, has nine keywords. The third most frequent keyword is "Severe Acute Respiratory Syndrome Coronavirus (SARSCOV)" with a frequency of 259 in this cluster. The key theme of this cluster is related to "Pathogenesis and SARS-COV". This cluster has seven keywords, and keywords such as Pathogenesis, Pathogenicity, Viral replication, and infection present in the $16^{\text {th }}$ cluster. By analyzing keywords, the main subject is "Pathogenesis of Viral Infections". The $17^{\text {th }}$ cluster, consist of six keywords. This study's most frequent keyword is "Severe Acute Respiratory Syndrome (SARS)", with a frequency of 276 in this cluster. Vaccine, the thirteenth high frequent keyword, is also in the cluster. "SARS and Vaccination" represent the central concept of the seventeenth cluster. The two keywords are "Monoclonal Antibody and Epitopes" in the $18^{\text {th }}$ cluster. So "Immunology and Coronavirus" is the main subject of this cluster. The $19^{\text {th }}$ cluster has six keywords, and Nucleocapsid protein is the ninth most frequent keyword in this cluster. $\mathrm{M}-\mathrm{CoV}$ is a type of Coronavirus that comes with the names of proteins. Hence the content of this cluster deals with the "structure of Coronavirus and Epidemic murine illness."

\section{Coronavirus Subject Clusters Density and Degree/Centrality}

The data in Table 3 showed the density and centrality of the nineteen Coronavirus subject clusters. Using UCINET software, the Degree / Centrality and Density indices of each cluster were calculated. Also, the average of these two indicators were calculated for all clusters. Clusters 11, 15, and 17 had the highest Degree / Centrality, respectively, and Categories 1, 5, and 11 had the higher densities than other clusters (Table 3).

\section{Coronavirus Strategic Diagram}

The Strategic Diagram's origin was determined by the Degree / Centrality and Density averages of the clusters. Degree / Centrality determined the interaction power of each cluster on the X-axis. The higher the centrality of a cluster is, the more critical and central it is. The internal relationship of the clusters with density in the Y-axis were also shown. The higher the density of a cluster is, the greater the cluster's ability is to maintain and develop $(13,33,34)$. A cluster located within a strategic diagram makes it characterize in the context of the whole discipline. In quadrant I; clusters were both coherent and centric to the field. These mainstream clusters figured the focus of a large section of the network. The quadrant II clusters were also 
coherent; however, they are likely to be specialized and separated from the overall focus. The clusters in quadrant III were in infusion, showing the emerging or declining sections of the network. Finally, quadrant IV contained the clusters that illustrate a common and broad focus or have not yet completed but have the potential to be considered as a primary network focus $(32,35)$. The data in Figure 3 shows that in quadrant I, cluster 11 ; in quadrant II, clusters $1,2,5$, and 6 ; in quadrant III, clusters $12,8,3,18,7,9,19$, 4, and 16; and in quadrant IV, clusters 15, 17, 10 and 13 are located.

Table 3. Coronavirus subject clusters density and Degree/ Centrality (1970-2019)

\begin{tabular}{|c|c|c|}
\hline Clusters & Degree/ Centrality & Density \\
\hline Cluster 1 & 1 & 18 \\
\hline Cluster 2 & 1 & 7 \\
\hline Cluster 3 & 1.91 & 2.6 \\
\hline Cluster 4 & 0.96 & 1.5 \\
\hline Cluster 5 & 1 & 11 \\
\hline Cluster 6 & 1.5 & 7 \\
\hline Cluster 7 & 1.08 & 1.1 \\
\hline Cluster 8 & 2 & 1.57 \\
\hline Cluster 9 & 1 & 2.66 \\
\hline Cluster 10 & 2.5 & 3.66 \\
\hline Cluster 11 & 11.5 & 7.6 \\
\hline Cluster 12 & 2.33 & 2.16 \\
\hline Cluster 13 & 2.5 & 2.66 \\
\hline Cluster 14 & 1 & 4 \\
\hline Cluster 15 & 5.14 & 1.5 \\
\hline Cluster 16 & 0.66 & 0.52 \\
\hline Cluster 17 & 4.6 & 2.33 \\
\hline Cluster 18 & 1.5 & 3 \\
\hline Cluster 19 & 1 & 1.53 \\
\hline Total & 44.18 & 81.39 \\
\hline Average & 2.32 & 4.28 \\
\hline
\end{tabular}

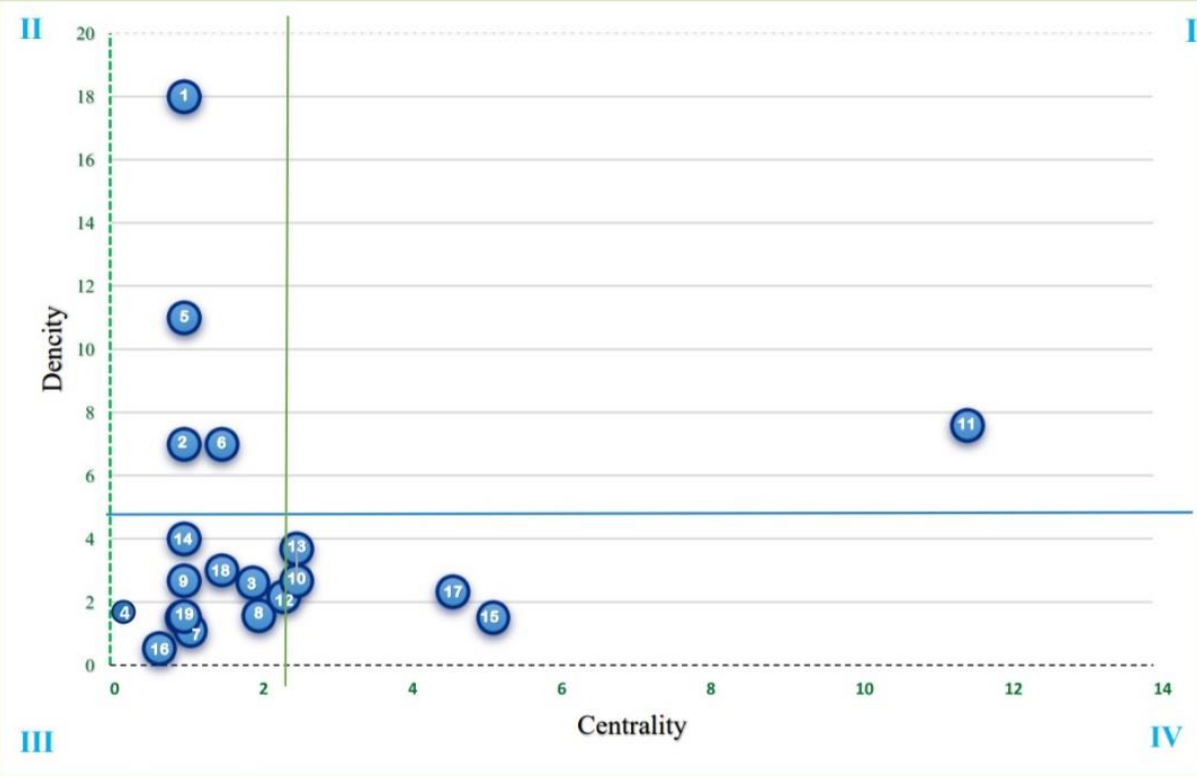

Figure 3. Coronavirus Strategic Diagram (1970-2019) 


\section{Discussion}

In this paper, using co-word analysis, social network analysis tools, and scientific visualization software, global knowledge structure, hidden patterns, and emerging events of Coronavirus in the last 50 years (1970 to 2019) were presented.

The findings showed that "Severe Acute Respiratory Syndrome (SARS)" has the highest frequency among Coronavirus keywords; besides, "Severe Acute Respiratory Syndrome CORONAVIRUS (SARSCOV)" is the third most frequent keyword. Therefore, SARS appears to have accounted for a large share of Coronavirus research before 2020. From November $1^{\text {st }}$, 2002 , to June $11^{\text {th }}, 2003$, SARS-CoV spread to thirty countries and infected 8098 people, and 916 ones lost their lives as a result of SARS-CoV. Its pandemic period lasted until 2004, so between 2002 and 2004, various aspects of the disease came to medical researchers' attention, and a large number of scientific publications were published on the importance of the SARS-CoV Outbreak (36).

"Middle East Respiratory Syndrome Coronavirus (MERS-COV)" is the second most frequent keyword; the newer Coronavirus, MERS-CoV, spread to Saudi Arabia in September 2012 (37). Outbreaks in twentyseven countries, infectious more than 2428 people, and the death of 838 were the effects of MERS-CoV. After Saudi Arabia, the disease recurred in the Republic of Korea in $2015(36,38,39)$. In order to identify, prevent, and treat MERS-CoV, medical scientists have conducted various studies that have led to the publication of significant scientific documents.

Examination of the results showed that the increase in Coronavirus's global scientific publications follows the outbreak of the new species of the virus, the pandemic stage, and the resulting death. So, with the onset of the massive COVID-19 epidemic, the latest form of Coronaviridae, and its imminent threat to global public health (40), we will see a dramatic increase in COVID-19 global science production soon.

In this paper, in addition to co-word analysis, the hierarchical cluster analysis method was also performed. The cluster analysis results led to the formation of 19 thematic Coronavirus clusters and identified the intellectual structure of Coronavirus research. The results of this article indicated that clusters such as "Coronavirus and the Peritonitis of infection," "Gastroenteritis and the transmission of crowned Coronavirus", "Turkey Bovine Coronavirus Immunohistochemistry", "Avian Coronavirus infectious bronchitis virus", "Human Coronavirus and viral infections", "Coronavirus Drug Therapy", "Coronavirus antibodies", "Outbreaks of zoonotic diseases caused by viruses MERS-COV", "Coronavirus infects in the CNS", "CoV life cycle", "Pathogenesis and SARS-COV", "Immunology and Coronavirus", and "structure of Coronavirus and
Epidemic murine illness" have been considered by medical researchers.

Tracking sciences illustrated Coronavirus research events' dynamics and the expression of hidden research patterns by identifying the structure, concepts, and components of Coronavirus outputs using the phonological analysis method. The results could be of great help to scientists and professionals who are currently researching COVID-19 to target research, given their research priorities.

The strategic diagram results in the present paper showed that the $11^{\text {th }}$ cluster "Outbreaks of zoonotic diseases caused by viruses MERS-COV" cluster has a high centrality and density. The $11^{\text {th }}$ cluster has a crucial role and is coherent and central. The topics "Coronavirus and the Peritonitis of infection", "Gastroenteritis and the transmission of crowned Coronavirus", "Avian Coronavirus infectious bronchitis virus," and "Human Coronavirus and viral infections," are also coherent but separate and do not play a central role. Subject clusters such as "Turkey Bovine Coronavirus Immunohistochemistry", "Coronavirus Laboratory Testing", "Molecular Epidemiology of Recombination Human Coronavirus", "Human Coronavirus and viral infections", "Coronavirus Drug Therapy", and "Pathogenesis of Viral Infections" have less centrality and density. They are of little importance in the field of coronavirus knowledge discovery. Given the prevalence of COVID-19 in the Coronaviridae family, these clusters represent emerging or declining portions of the network. It is essential to consider such concepts. Understanding the structure and characteristics of COVID-19 will allow specialist physicians to discover new vaccines, medications, and novel treatments. The thematic clusters "Coronavirus antibodies", "Structures and Mechanisms of Coronavirus Cell", "Pathogenesis and SARS-COV", and "SARS and Vaccination" have high centrality and low density. The clusters have not yet matured but can be a primary network focus (32).

The present study complements the novel Coronavirus (2019-nCoV) (18). Danesh and Ghavidel's research used scientometric indicators to represent the quantitative and qualitative content of coronavirus scientific publications. The results of both studies showed that the amount of coronavirus outbreak in different periods has led to the growth of scientific publications in the Coronavirus subject domain. Also, this paper's results are in line with the studies of Bonilla-Aldana et al. and (18) and Zyoud (21). In analyzing and identifying the connections between the concepts of a thematic domain in medical sciences, the present study is consistent with the Human Papilloma Virus (19). In that study, the concepts were analyzed in the form of thematic clusters, and the maturity and coherence of the topics were presented using the strategic diagram (19). According to research on the subject matter of viruses $(21-24,41)$, various and varied scientometrics methods have been used to analyze each 
topic. Important and strategic results obtained from scientometric studies, especially from the present research, make researchers and specialists in respiratory and infectious diseases familiar with the coronavirus's knowledge structure and thematic contexts. Research policy-makers can also use the results of the paper obtained in macro-planning.

The authors of the paper believe that it is necessary to conduct co-citation and co-authorship research to identify the citation network, introduce proliferated scientists, and discover global scholarly collaboration patterns. Since the outbreak of COVID-19 numerous articles were published; we suggest that all the COVID-19' s publications in 2020 be analyzed in an independent research using scientometric indicators. Undoubtedly, the results of such studies will be of interest to international medical researchers and policymakers.

\section{Conclusion}

Discovering the structure of global knowledge and its impact on the research of the Coronavirus will establish a link between human knowledge and the activities of scientists and specialists during the COVID-19 pandemic. Representing and visualizing Coronavirus's scientific structure, using hierarchy clustering, showed the breadth of concepts and the discovery of the hidden connections and patterns of Coronavirus. The maturity and cohesion of each of the Coronavirus clusters will guide Coronavirus researchers toward emerging events and pave the way for planners and policymakers in international medical organizations.

\section{Ethical Considerations}

Ethical issues (Including plagiarism, informed consent, misconduct, data fabrication and/or falsification, double publication and/or submission, redundancy, etc.) have been completely observed by the authors.

\section{Acknowledgments}

The authors of this article would like to appreciate the specialists in respiratory and infectious diseases for expressing valuable views and comments.

\section{Conflict of Interest}

Authors declared no conflict of interest.

\section{References}

1. Jalali Nadoushan M, Ahmadi S, Jalali Nadoushan P. Serology Testing for SARS-CoV2: Benefits and Challenges. Iranian Journal of
Pathology. 2020;15(3):154-5. [DOI:10.30699/ijp.2020.39841] [ [PMCID]

2. Wen J, Aston J, Liu X, Ying T. Effects of misleading media coverage on public health crisis: a case of the 2019 novel coronavirus outbreak in China. Anatolia. 2020;31(2):331-6. [DOI:10.1080/13032917.2020.1730621]

3. Lai C-C, Shih T-P, Ko W-C, Tang H-J, Hsueh PR. Severe acute respiratory syndrome coronavirus 2 (SARS-CoV-2) and coronavirus disease-2019 (COVID-19): The epidemic and the challenges. International Journal of Antimicrobial Agents. 2020;55(3):105924. [DOI:10.1016/j.ijantimicag.2020.105924] [PMID] [PMCID]

4. Sakurai A, Sasaki T, Kato S, Hayashi M, Tsuzuki $\mathrm{S}-\mathrm{i}$, Ishihara $\mathrm{T}$, et al. Natural History of Asymptomatic SARS-CoV-2 Infection. New England Journal of Medicine. 2020. [DOI:10.1056/NEJMc2013020] [ [PMCID]

5. Zhu Z, Zhang Z, Chen W, Cai Z, Ge X, Zhu H, et al. Predicting the receptor-binding domain usage of the Coronavirus based on kmer frequency on spike protein. Infect Genet Evol. 2018;61:183-4.

[DOI:10.1016/j.meegid.2018.03.028] [ [PMID] [PMCID]

6. Fehr AR, Perlman S. Coronaviruses: an overview of their replication and pathogenesis. Methods Mol Biol. 2015;1282:1-23. [DOI:10.1007/978-1-4939-2438-7_1] [PMID] [PMCID]

7. Paraskevis D, Kostaki EG, Magiorkinis G, Panayiotakopoulos G, Sourvinos G, Tsiodras S. Full-genome evolutionary analysis of the novel corona virus (2019-nCoV) rejects the hypothesis of emergence as a result of a recent recombination event. Infect Genet Evol. 2020;79:104212.

[DOI:10.1016/j.meegid.2020.104212] [PMID] [PMCID]

8. Cui J, Li F, Shi Z-L. Origin and evolution of pathogenic coronaviruses. Nature Reviews Microbiology. 2019;17(3):181-92. [DOI:10.1038/s41579-018-0118-9] [피] [PMCID]

9. Phan T. Novel coronavirus: From discovery to clinical diagnostics. Infect Genet Evol. 2020;79:104211.

[DOI:10.1016/j.meegid.2020.104211] [PMID] [PMCID]

10. CSSE J. Coronavirus COVID-19 Global Cases by the Center for Systems Science and 
Engineering (CSSE) at Johns Hopkins University (JHU). 2020.

11. Birkle C, Pendlebury DA, Schnell J, Adams J. Web of Science as a data source for research on scientific and scholarly activity. Quantitative Science Studies. 2020;1(1):363-76. [DOI:10.1162/qss_a_00018]

12. Niloofar $\mathrm{P}$, Hassan $\mathrm{H}$, Gholamreza A, Mohammadhadi A, Ghasem J, Reza M, et al. Scientometric Study on Non-communicable Diseases in Iran: A Review Article. Iranian Journal of Public Health. 2018;47(7).

13. Liu G-Y, Hu J-M, Wang H-L. A co-word analysis of digital library field in China. Scientometrics. 2012;91(1):203-17. [DOI:10.1007/s11192-011-0586-4]

14. Chen X, Chen J, Wu D, Xie Y, Li J. Mapping the Research Trends by Co-word Analysis Based on Keywords from Funded Project. Procedia Computer Science. 2016;91:547-55. [DOI:10.1016/j.procs.2016.07.140]

15. Lee $\mathrm{P}-\mathrm{C}, \mathrm{Su} \mathrm{H}-\mathrm{N}$. Investigating the structure of regional innovation system research through keyword co-occurrence and social network analysis. Innovation. 2010;12(1):26-40. [DOI:10.5172/impp.12.1.26]

16. Ravikumar S, Agrahari A, Singh SN. Mapping the intellectual structure of scientometrics: a coword analysis of the journal Scientometrics (2005-2010). Scientometrics. 2015;102(1):92955. [DOI:10.1007/s11192-014-1402-8]

17. Danesh F, Ghavidel S. Coronavirus: Scientometrics of 50 Years of Global Scientific Productions. Iran J Med Microbiol. 2020;14(1):1-16. [DOI:10.30699/ijmm.14.1.1]

18. Bonilla-Aldana DK, Quintero-Rada K, Montoya-Posada JP, Ramirez-Ocampo S, PanizMondolfi A, Rabaan AA, et al. SARS-CoV, MERS-CoV and now the 2019-novel CoV: Have we investigated enough about coronaviruses? - A bibliometric analysis. Travel Med Infect Dis. 2020;33:101566.

[DOI:10.1016/j.tmaid.2020.101566] [PMID] [PMCID]

19. Danesh F, Ghavidel S. Visualizing the Clusters and Dynamics of HPV Research Area. Iranian Journal of Medical Microbiology. 2019;13(4):266-78.

[DOI:10.30699/ijmm.13.4.266]

20. Singh N, Brar R, Chavan SB, Singh J. Scientometric analyses and visualization of a scientific outcome on the Nipah virus. CURRENT SCIENCE (A Fortnightly Journal of Research). 2019;117(10):1574-84 [DOI:10.18520/cs/v117/i10/1574-1584]
21. Zyoud SH. Global research trends of Middle East respiratory syndrome coronavirus: a bibliometric analysis. BMC Infect Dis. 2016;16:255. [DOI:10.1186/s12879-016-1600-5] [PMID] [PMCID]

22. Chiu WT, Huang JS, Ho YS. Bibliometric analysis of Severe Acute Respiratory Syndromerelated research in the beginning stage. Scientometrics. 2004;61(1):69-77. [DOI:10.1023/B:SCIE.0000037363.49623.28] [PMID] [PMCID]

23. Ekundayo TC, Okoh AI. A global bibliometric analysis of Plesiomonas-related research (1990 2017). PLoS One. 2018;13(11):e0207655. [DOI:10.1371/journal.pone.0207655] [PMID] [PMCID]

24. Khazaneha M, Heaidary G, Mostafavi I. Structural analyzing of Information Science Theories based on co-word network analysis of articles in Web of Science database (1983-2017). Iranian Journal of Processing and Management. 2019;34(3):1051-76.

25. Ahmadi H, Osareh F. Co-word analysis concept, definition and application. 2017.

26. Analytics C. Web of science platform: Web of science: Summary of coverage. 2018.

27. Hu C-P, Hu J-M, Deng S-L, Liu Y. A co-word analysis of library and information science in China. Scientometrics. 2013;97(2):369-82. [DOI:10.1007/s11192-013-1076-7]

28. Lee B, Jeong Y-I. Mapping Korea's national $\mathrm{R} \& \mathrm{D}$ domain of robot technology by using the co-word analysis. Scientometrics. 2008;77(1):319. [DOI:10.1007/s11192-007-1819-4]

29. Neff MW, Corley EA. 35 years and 160,000 articles: A bibliometric exploration of the evolution of ecology. Scientometrics. 2009;80(3):657-82. [DOI:10.1007/s11192-0082099-3]

30. Ding Y, Chowdhury GG, Foo S. Bibliometric cartography of information retrieval research by using co-word analysis. Information Processing \& Management. 2001;37(6):817-42. [DOI:10.1016/S0306-4573(00)00051-0]

31. Zong Q-J, Shen H-Z, Yuan Q-J, Hu X-W, Hou Z-P, Deng S-G. Doctoral dissertations of Library and Information Science in China: A co-word analysis. Scientometrics. 2013;94(2):781-99. [DOI:10.1007/s11192-012-0799-1]

32. Melcer E, Nguyen T-HD, Chen Z, Canossa A, El-Nasr MS, Isbister K. Games research today: Analyzing the academic landscape 2000-2014. network. 2015;17:20.

33. Law J, Bauin S, Courtial JP, Whittaker J. Policy and the mapping of scientific change: A co-word 
analysis of research into environmental acidification. Scientometrics. 1988;14(3):25164. [DOI:10.1007/BF02020078]

34. Sohaili F, Shaban A, Khase A. Intellectual structure of knowledge in information behavior: A co-word analysis. Human Information Interaction. 2016;2(4):21-36.

35. Giannakos M, Papamitsiou Z, Markopoulos P, Read J, Hourcade JP. Mapping child-computer interaction research through co-word analysis. International Journal of Child-Computer Interaction. 2020;23-24:100165. [DOI:10.1016/j.ijcci.2020.100165]

36. Song Z, Xu Y, Bao L, Zhang L, Yu P, Qu Y, et al. From SARS to MERS, Thrusting Coronaviruses into the Spotlight. Viruses. 2019;11(1). [DOI:10.3390/v11010059] [PMID] [PMCID]

37. Al-Tawfiq JA, Gautret P. Asymptomatic Middle East Respiratory Syndrome Coronavirus (MERS-CoV) infection: Extent and implications for infection control: A systematic review. Travel Med Infect Dis. 2019;27:27-32. [DOI:10.1016/j.tmaid.2018.12.003] [PMID] [PMCID]
38. Momattin H, Al-Ali AY, Al-Tawfiq JA. A Systematic Review of therapeutic agents for the treatment of the Middle East Respiratory Syndrome Coronavirus (MERS-CoV). Travel Med Infect Dis. 2019;30:9-18. [DOI:10.1016/j.tmaid.2019.06.012] [ [PMID] [PMCID]

39. Leist SR, Jensen KL, Baric RS, Sheahan TP. Increasing the translation of mouse models of MERS coronavirus pathogenesis through kinetic hematological analysis. PLoS One. 2019;14(7):e0220126.

[DOI:10.1371/journal.pone.0220126] [PMID] [PMCID]

40. Abdollahi A, Mahmoudi-aliabadi M, Mehrtash V, Jafarzadeh B, Salehi M. The Novel Coronavirus SARS-CoV-2 Vulnerability Association with ABO/Rh Blood Types. Iranian Journal of Pathology. 2020;15(3):156-60. [DOI:10.30699/ijp.2020.125135.2367] [PMID] [PMCID]

41. Danesh F, Ghavidel S. Coronavirus: Scientometrics of 50 Years of Global Scientific Productions. Iranian Journal of Medical Microbiology. 2020;14(1):1-16. [DOI:10.30699/ijmm.14.1.1]

\section{How to Cite This Article:}

Danesh F, GhaviDel S, Piranfar V. Coronavirus: Discover the Structure of Global knowledge, Hidden Patterns \& Emerging events. J Adv Med Biomed Res. 2020; 28 (130) :253-264

\section{Download citation:

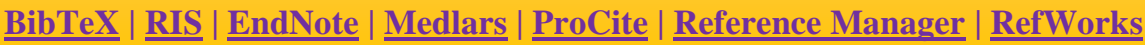 \\ Send citation to: \\ Mendeley 2 Zotero (i) RefWorks RefWorks}

